Ann. Zootech., I966, 15 (3), 279-290.

\title{
ÉTUDE DE LA RÉPARTITION TOPOGRAPHIQUE DE LA MYOPATHIE EXSUDATIVE ET DÉPIGMENTAIRE DU PORC
}

\author{
R. GOUTEFONCiEA et J. CHARPENTIER \\ avec la collaboration technique de Denise Gizene et J.-l'. Siquet \\ Laboratoire de Recherches sur la Viande, \\ Centre national de Recherches sootechniques, 78-Jouy-en-Josas
}

\section{SOMMAIRE}

Trente demi-carcasses de porcs Large White abattus au poids vif de $100: \pm 5 \mathrm{~kg}$ ont été disséquées le lendemain de l'abattage. Pour chaque demi-carcasse, 5 I échantillons, représentant 45 muscles, ont été soumis à la détermination du $\mathrm{pH}$, du pourcentage de rémission et du pouvoir de rétention d'eau.

Dans toute carcasse de porc, certains muscles ont toujours un pH ultime faible, un pourcentage de rémission élevé (coloration pâle) et un pouvoir de rétention d'eau faible. De plus, dans ces muscles, la variabilité des caractéristiques mesurées est plus élevée. Lorsque l'on utilise la mesure d'une ou plusieurs de ces caractéristiques pour apprécier objectivement la qualité d'une carcasse, on a donc intérêt, pour augmenter la sensibilité, à s'adresser aux muscles pâles et à faible pouvoir de rétention d'eau.

De nombreux travaux sont consacrés, depuis plus de io ans, à l'étude de l'affection connue sous le nom de myopathie exsudative et dépigmentaire du porc (COSSARD, I957) en France, et de watery pork dans les pays anglo-saxons. Diverses revues bibliographiques ont, par ailleurs, fait le point sur l'ensemble de ces travaux (GoUTEFONGEA, I963), (BENDAIL et LAWRIE, I964), (BRISKEY, I964).

Il est reconnu que dans cette affection, il existe une continuité entre les caractéristiques physiques et physico-chimiques de la musculature de 1'animal parfaitement normal et celles de l'animal profondément atteint, mais on sait également que dans un animal, tous les muscles ne sont pas atteints avec la même intensité. Une étude préliminaire (MESLE et $a l$., I960), effectuée sur 5 muscles du membre postérieur du porc, nous a permis d'observer une importante variabilité des critères pris en considération, à savoir le $\mathrm{pH}$, le pouvoir de rétention d'eau et la couleur, ainsi que l'existence de liaisons entre ces critères. 
Bien que le membre postérieur représente une part importante de la valeur de la carcasse, nous avons estimé nécessaire d'étendre cette étude à l'ensemble de la musctlature et ceci dans un double but :

- vérifier la validité de nos observations effectuées sur un nombre réduit de muscles provenant d'une même région anatomique,

- étudier la répartition topographique de l'affection et déterminer les muscles les plus sensibles.

\section{MATÉRIEL ET MÉTHODES}

\section{Matériel animal}

Trente porcs de race Large White pesant too + $5 \mathrm{~kg}$ ont été utilisés lors de cette étude.

Les opérations d'abattage comprenaient successivement l'électrocution, la saignée, l'échaudage, l'éviscération et la fente.

Les carcasses des porcs étaient placées en chambre froide à $+4^{\mathrm{o}}, 2$ heures après la mort. La dissection (sur une demi-carcasse) avait lieu le lendemain. Nous avons pris en considération tous les muscles qui, par leur taille et leur structure, permettaient d'effectuer des mesures dans de bonnęs conditions. Nous avons dû en effet éliminer certains muscles ou trop petits, ou constamment infiltrés de graisse et de tissu conjonctif, ce qui ne nous aurait pas permis d'obtenir des résultats valables, en ce qui concerne la mesure du $\mathrm{pH}$, de la couleur ou du pouvoir de rétention d'eau.

Pour certains muscles, présentant manifestement deux zones de coloration très différente, nous avons considéré deux échantillons par muscle.

Nous avons ainsi pu étudier 45 muscles représentés par $5 \mathrm{I}$ échantillons dont la liste est donnée en annexe. La nomenclature de ces muscles a été effectuée selon Nickel, Schummer et SEIfERLE (1954).

\section{Méthodes}

Un échantillon d'un poids minimum de $25 \mathrm{~g}$ était prélevé sur chaque muscle ou partie de muscle en s'attachant à ce qu'il soit le plus homogène possible et exempt de gras ou d'aponévrose.

Sur chaque échantillon, les déterminations suivantes étaient effectuées :

a) Mesure đu pourcentage de rémission à $490 \mathrm{~m} u$ à l'aide d'un réflectomètre électrosynthèse $\mathrm{SP}_{3}$ (mesure du pourcentage de lumière incidente réfléchie par l'échantillon). Plus un muscle est clair, plus ce pourcentage de rémission est élevé.

b) Mesure du pII au moyen d'un pH-mètre EIL ${ }_{2} 3 \mathrm{AF}_{2}$ et d'une électrode de type duplex $\mathrm{SDSA}_{30}$.

c) Mesure du $\mathrm{pH}$ sur le broyat obtenu à l'aide d'un hachoir muni d'une grille à trous de $3 \mathrm{~mm}$ de diamètre.

d) Détermination du pouvoir de rétention d'eau par la méthode par pression décrite par GouTEFONGEA (1966). A l'issue de cette mesure, on traduisait le pouvoir de rétention d'eau par le pourcentage d'eau défini par :

$$
\text { P. Ioo eau libre }=\frac{\text { Poids initial de l'échantillon }- \text { poids final }}{\text { Poids initial }} \times 100
$$

Donc plus un muscle a un pouvoir de rétention d'eau faible, plus le pourcentage d'eau libre est élevé.

\section{RÉSULTATS - DISCUSSION}

Nous avons rassemblé, dans les tableaux I, 2, 3 et 4 , les valeurs moyennes $\bar{x}$, les écarts-types $\sigma$ et les coefficients de variation $\frac{\sigma}{\overline{\bar{x}}}$ des caractères mesurés pour chaque échantillon. Nous les avons classés dans l'ordre des valeurs décroissantes pour la 


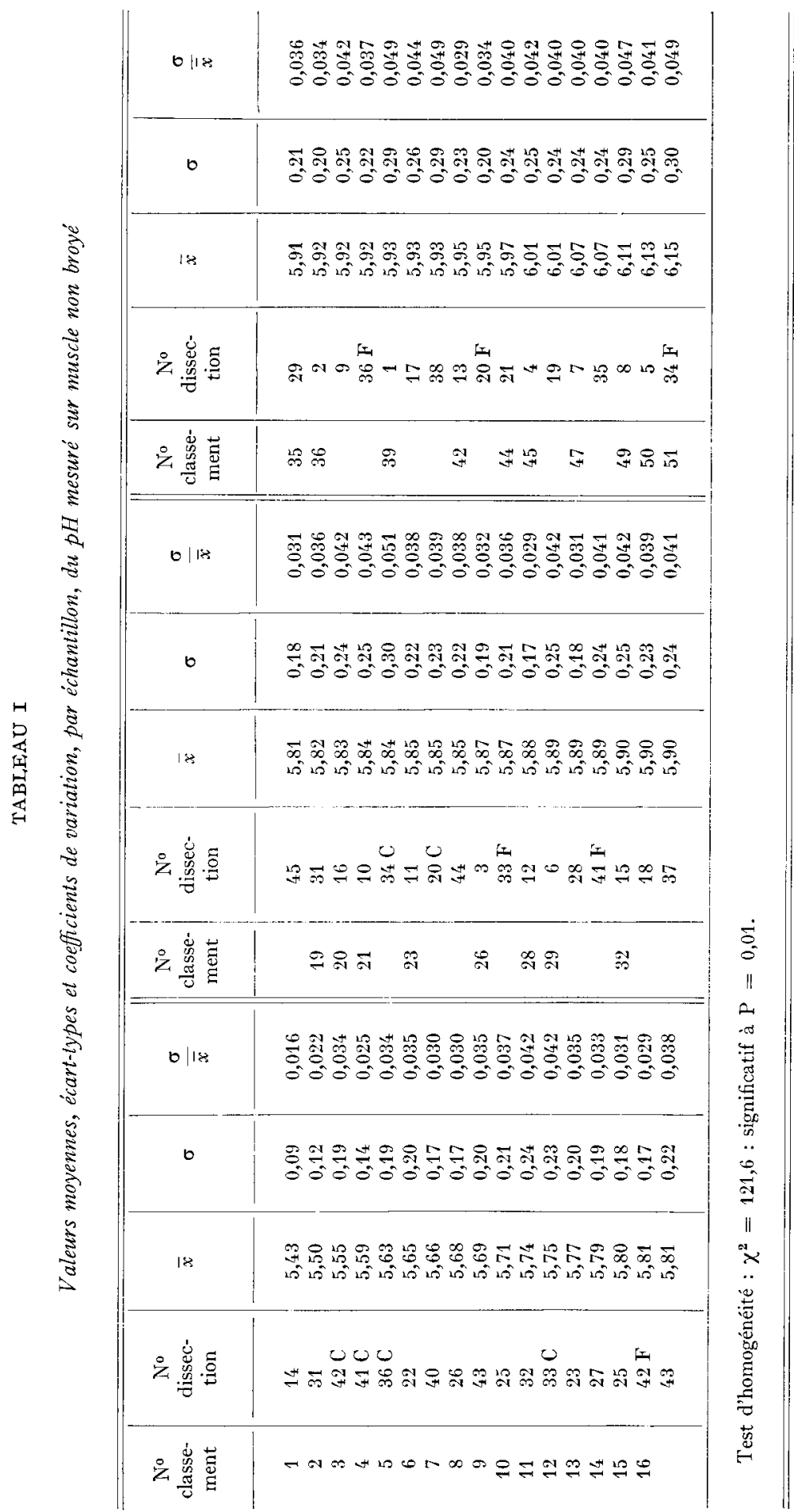


R. GOUTEFONGEA, J. CHARPENTIER

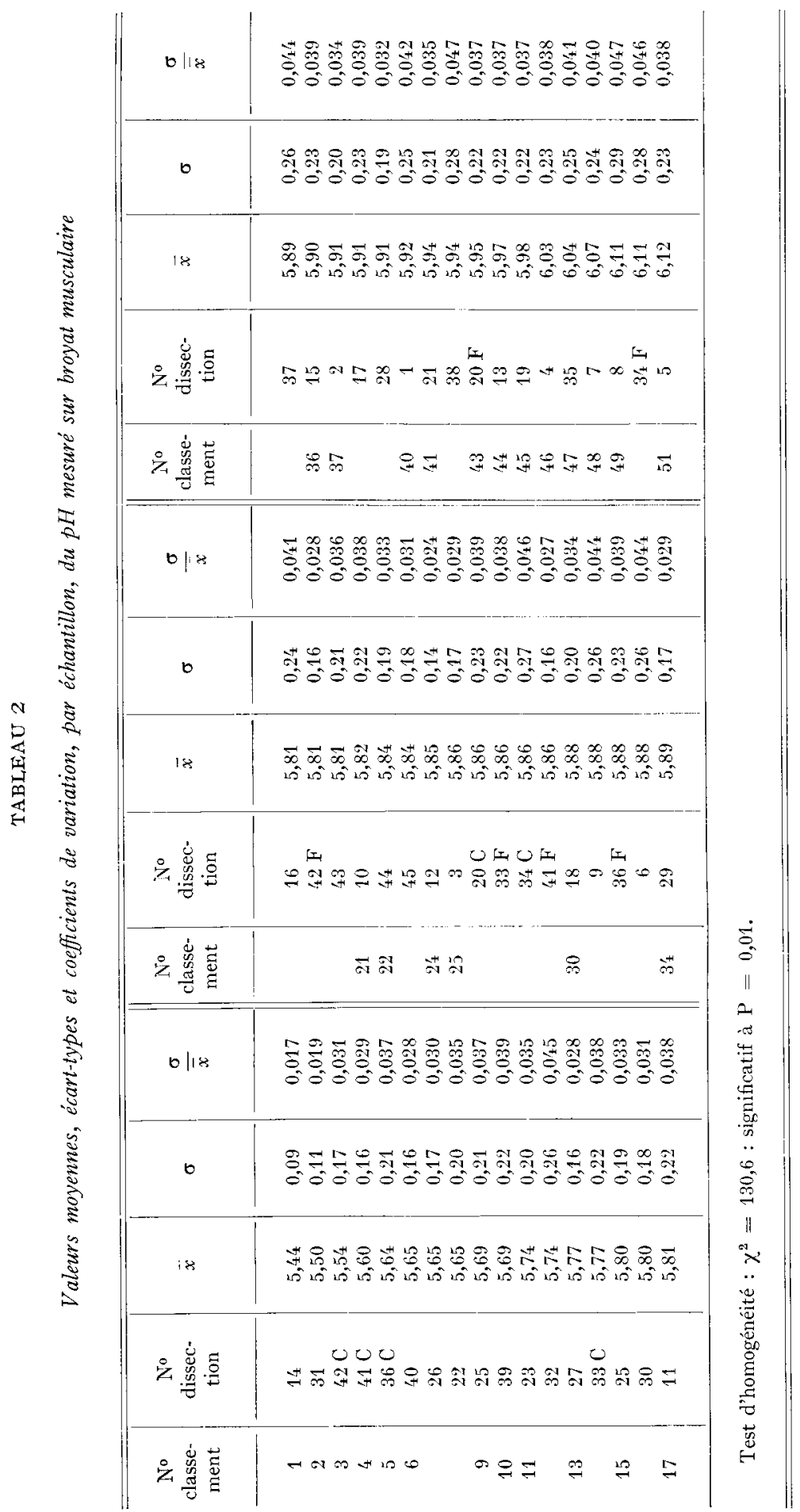




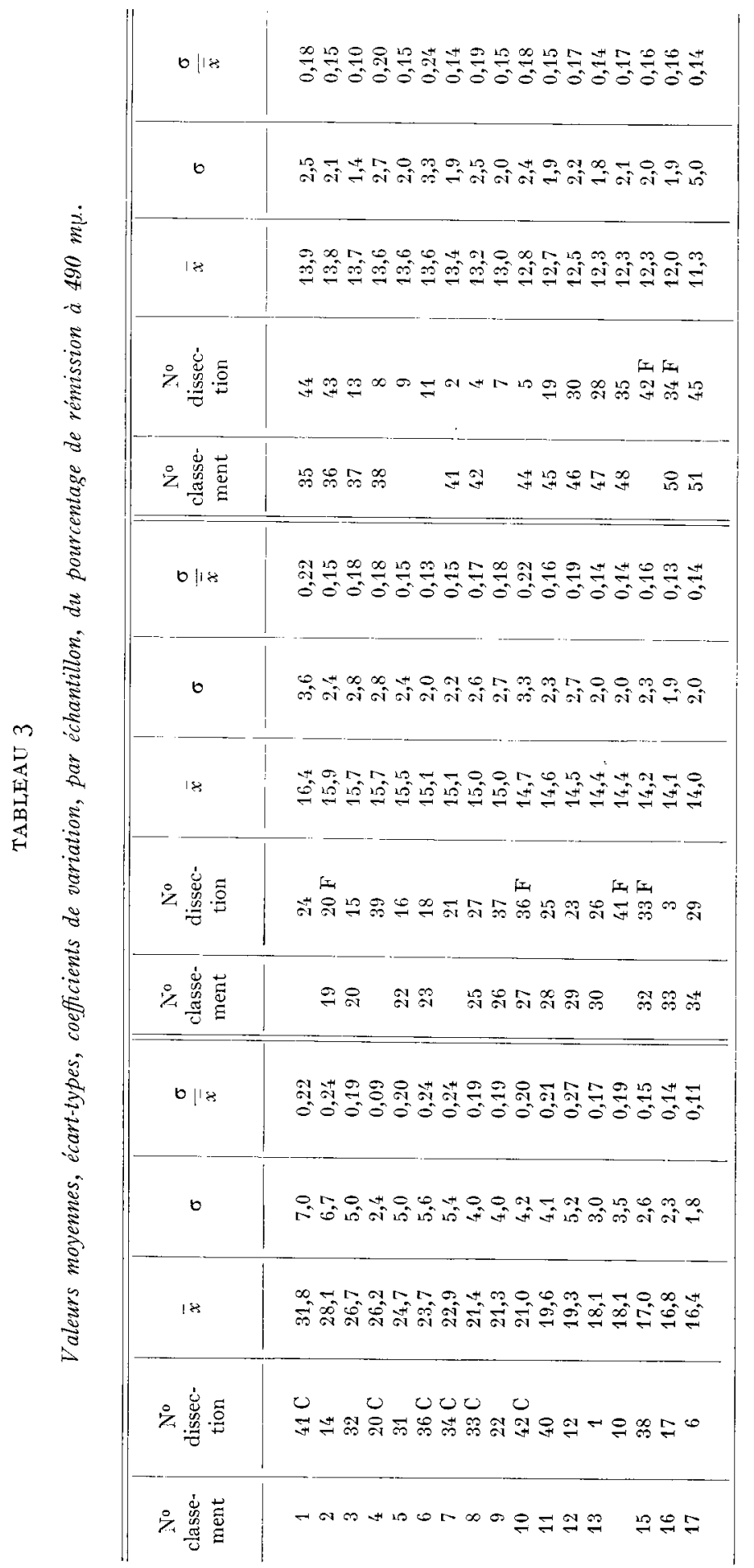


R. GOUTEFONGEA, J. CHARPENTIER

\begin{tabular}{|c|c|c|}
\hline & 0118 & 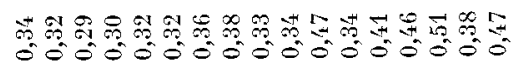 \\
\hline & o & 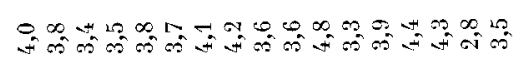 \\
\hline కี้ & 18 & 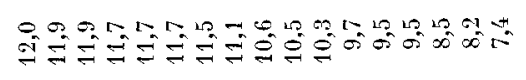 \\
\hline (气) & 悹兽 & 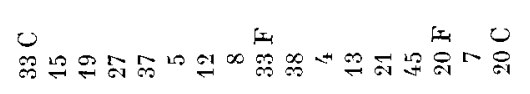 \\
\hline & 象离蒙 & 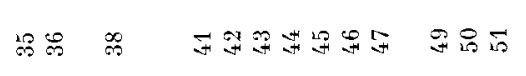 \\
\hline & ب & 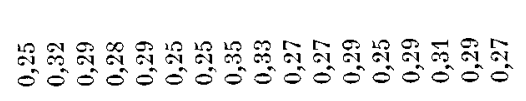 \\
\hline & ○ & 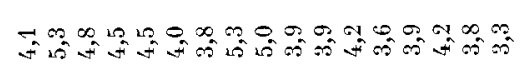 \\
\hline 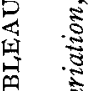 & 18 & 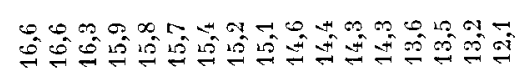 \\
\hline$\frac{\mathrm{g}}{\mathrm{s}}$ & 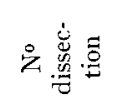 & 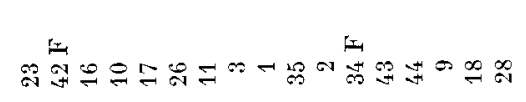 \\
\hline 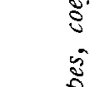 & 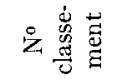 & 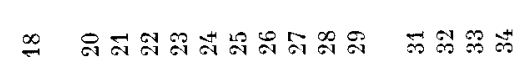 \\
\hline 莣 & $0 \| 18$ & 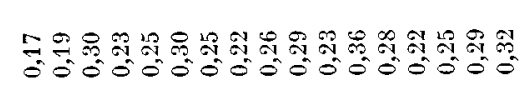 \\
\hline 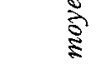 & ○ & 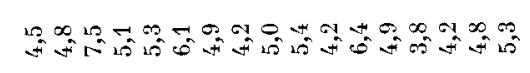 \\
\hline$\dot{A}$ & 18 & 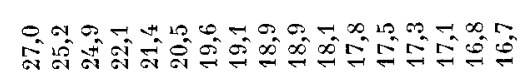 \\
\hline & 离总苟 & 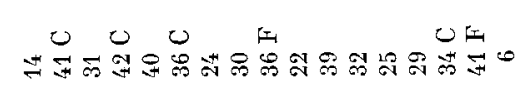 \\
\hline & 号离蓄 & - \\
\hline
\end{tabular}


Coefficient de corrélation par échantillon entre les 4 déterminations effectuées

Détermination I : $\mathrm{pH}$ mesuré sur échantillon entier

Détermination $2: \mathrm{pH}$ mesuré sur broyat

Détermination 3 : Pourcentage de rémission

Détermination 4 : Pouvoir de rétention d'eau

\begin{tabular}{|c|c|c|c|c|c|c|}
\hline $\mathrm{N}^{\circ}$ & $1 / 2$ & $1 / 3$ & $1 / 4$ & $2 / 3$ & $2 / 4$ & $3 / 4$ \\
\hline 1 & $+0,96 * *$ & $-0,53 * *$ & $-0,49 * *$ & $-0,51 * *$ & $-0,49 * *$ & $+0,23$ \\
\hline 2 & $+0,90 * *$ & $-0,59 * *$ & $-0,55 * *$ & $-0,38 *$ & $-0,59 * *$ & $+0,26$ \\
\hline 3 & $+0,88^{* *}$ & $-0,21$ & $-0,38 * *$ & $-0,24$ & $-0,28$ & $-0,25$ \\
\hline 4 & $+0,97 * *$ & $-0,15$ & 一 $0,39 *$ & $-0,16$ & $-0,39 *$ & $+0,08$ \\
\hline 5 & $+0,92 * *$ & $-0,49 * *$ & $-0,75 * *$ & $-0,53 * *$ & $-0,69 * *$ & $+0,33$ \\
\hline 6 & $+0,97 * *$ & $-0,41 *$ & $-0,71 * *$ & $-0,32$ & $-0,76^{* *}$ & $+0,17$ \\
\hline 7 & $+0,96 * *$ & $-0,43^{*}$ & $-0,73^{* *}$ & $-0,32$ & $\cdots 0,71 * *$ & $+0,30$ \\
\hline 8 & $+0,96^{* *}$ & $-0,26$ & $-0,61 * *$ & $\rightarrow 0,26$ & $-0,58 * *$ & $+0,54 * *$ \\
\hline 9 & $+0,96 * *$ & $-0,43^{*}$ & $-0,10 * *$ & $-0,44^{*}$ & $-0,71 * *$ & $+0,10$ \\
\hline 10 & $+0,96 * *$ & $-0,59 * *$ & $-0,45^{* *}$ & - $0,56 * *$ & $-0,50 * *$ & $+0,26$ \\
\hline 11 & $+0,96 * *$ & $-0,51 * *$ & $-0,71 * *$ & $-0,46 * *$ & $-0,67 * *$ & $+0,48 * *$ \\
\hline 12 & $+0,91 * *$ & $+0,11$ & $+0,13$ & $-0,05$ & $-0,27$ & $+0,19$ \\
\hline 13 & $+0,97 * *$ & $-0,26$ & $-0,54 * *$ & $-0,23$ & $-0,56 * *$ & $-0,07$ \\
\hline 14 & $+0,74 *$ & $+0,11$ & $+0,09$ & $+0,12$ & $+0,10$ & $+0,37^{*}$ \\
\hline 15 & $+0,94 * *$ & $-0,42 \%$ & $-0,67 * *$ & $-0,35 *$ & $-0,71 * *$ & $+0,26$ \\
\hline 16 & $+0,97 * *$ & $-0,52 * *$ & $-0,62 * *$ & $-0,49 * *$ & $-0,60 * *$ & $+0,34$ \\
\hline 17 & $+0,96 * *$ & $-0,38 *$ & $-0,62 * *$ & $-0,34$ & $-0,63^{* *}$ & $+0,29$ \\
\hline 18 & $+0,95 * *$ & $-0,24$ & $-0,33$ & $-0,20$ & $-0,36 *$ & $+0,08$ \\
\hline 19 & $+0,96 * *$ & $-0,36 *$ & $-0,11 *$ & $-0,34$ & $-0,46^{* *}$ & $+0,16$ \\
\hline $20 \mathrm{~F}$ & $+0,97 * *$ & $-0,16$ & $-0,36$ * & $-0,19$ & $-0,43 *$ & $+0,47 * *$ \\
\hline $20 \mathrm{C}$ & $+0,96 * *$ & $-0,32$ & $-0,37 *$ & $-0,30$ & $-0,42 *$ & $+0,03$ \\
\hline 21 & $+0,94 * *$ & $-0,33$ & $-0,45 * *$ & $-0,38 *$ & $-0,44 *$ & $\begin{array}{r}1 \\
+0,27\end{array}$ \\
\hline 22 & $+0,92 * *$ & $-0,36 *$ & $-0,41 *$ & $-0,36 *$ & $-0,52 * *$ & $+0,35 *$ \\
\hline 23 & $+0,95 * *$ & $-0,25$ & $-0,07$ & $-0,29$ & $-0,06$ & $+0,04$ \\
\hline $2 / \mathbf{k}$ & $+0,97 *$ & $-0,36 *$ & $-0,60 * *$ & $-0,30$ & $-0,58 * *$ & $+0,16$ \\
\hline 25 & $+0,95 * *$ & $-0,13$ & $-0,47 * *$ & $-0,06$ & $-0,50^{* *}$ & $-0,01$ \\
\hline 26 & $+0,95^{* *} *$ & $-0,37 *$ & $-0,23$ & $-0,34$ & $-0,23$ & $+0,25$ \\
\hline 27 & $+0,87^{* *}$ & $+0,01$ & $-0,56 * *$ & $+0,01$ & $-0,50 * *$ & $\begin{array}{r}+ \\
+\end{array}$ \\
\hline 28 & $+0,95 * *$ & $-0,38 *$ & $-0,61 * *$ & $-0,34$ & $-0,65 *$ & $+0,30$ \\
\hline 29 & $+0,96^{* *}$ & $-0,48 * *$ & $-0,45 * *$ & $-0,46^{* *}$ & $-0,50 * *$ & $\begin{array}{r}0,27 \\
+\end{array}$ \\
\hline 30 & $+0,95 * *$ & $-0,36 *$ & $-0,62 * *$ & $-0,33$ & $-0,70 * *$ & $+0,30$ \\
\hline 31 & $+0,92 * *$ & $+0,15$ & $-0,18$ & $+0,07$ & $-0,11$ & $+0,42 *$ \\
\hline 32 & $+0,97 * *$ & $-0,5 \mathbf{4} * *$ & $-0,42 *$ & $-0,50 * *$ & $-0,43 *$ & $+0,43 *$ \\
\hline $33 \mathrm{~F}$ & $+0,98 * *$ & $-0,43^{*}$ & $-0,50 * *$ & $-0,43^{*}$ & $-0,5$ 五 $*$ & $+0,32$ \\
\hline $33 \mathrm{C}$ & $+0,95^{* *}$ & $-0,30$ & $-0,44^{*}$ & $-0,29$ & $-0,47^{*}$ & $+0,25$ \\
\hline $34 \mathrm{~F}$ & $+0,96 * *$ & $-0,35^{*}$ & $-0,70 * *$ & $-0,34$ & $-0,75 *$ & $+0,50 * *$ \\
\hline $34 \mathrm{C}$ & $+0,95 *$ & $-0,69 * *$ & $-0,69 * *$ & 一 $0,64 * *$ & $-0,71 * *$ & $+0,63^{* *}$ \\
\hline 35 & $+0,95 * *$ & $-0,25$ & $-0,59 * *$ & $-0,14$ & $-0,63^{* *}$ & $+0,37 *$ \\
\hline $36 \mathrm{~F}$ & $+0,92 * *$ & $-0,37 *$ & $-0,63 * *$ & $-0,31$ & $-0,62 * *$ & $+0,62 *$ \\
\hline $36 \mathrm{C}$ & $+0,82 * *$ & $-0,42 *$ & $-0,37 *$ & $-0,42 *$ & $-0,23$ & $-0,07$ \\
\hline 37 & $+0,95 * *$ & $-0,07$ & $-0,40^{*}$ & $-0,23$ & $-0,45^{* *}$ & $+0,50 * *$ \\
\hline 38 & $+0,99 * *$ & $-0,39 *$ & $-0,73^{* *}$ & $-0,4^{*} *$ & $-0,75 * *$ & $+0,24$ \\
\hline 39 & $+0,97 * *$ & $-0,45 * *$ & $-0,62 * *$ & $-0,43 *$ & $-0,60^{* *}$ & $+0,42 *$ \\
\hline 40 & $+0,96 * *$ & $-0,41 *$ & $-0,1^{\prime} *$ & $-0,38 *$ & $-0,40^{*}$ & $+0,22$ \\
\hline $41 \mathrm{~F}$ & $+0,97 * *$ & $-0,46 * *$ & $-0,68^{* *}$ & $-0,40^{*}$ & $-0,62 * *$ & $+0,36^{*}$ \\
\hline $41 \mathrm{C}$ & $+0,90 * *$ & $-0,17$ & $-0,07$ & $-0,22$ & $-0,28$ & $+0,23$ \\
\hline $42 \mathrm{~F}$ & $+0,81 * *$ & $-0,31$ & $-0,37 *$ & $-0,11$ & $-0,48 * *$ & $-0,05$ \\
\hline $42 \mathrm{C}$ & $+0,96 * *$ & $-0,36 *$ & $-0,34$ & $-0,25$ & $-0,26$ & $+0,46^{* *}$ \\
\hline 43 & $+0,96 * *$ & $-0,07$ & $-0,54 * *$ & $-0,02$ & $-0,52 * *$ & $+0,39 *$ \\
\hline 44 & $+0,914 *$ & $-0,0^{\prime}$ & $-0,56 * *$ & $+0,01$ & $-0,47 * *$ & $+0,61 * *$ \\
\hline 45 & $+0,90 * *$ & $-0,09$ & $-0,50^{* *}$ & $+0,05$ & $-0,49 * *$ & $\therefore 0,01$ \\
\hline
\end{tabular}

$*$ significatif à $\mathrm{P}=0,05 . \quad * *=$ significatif à $\mathrm{P}=0,01$.

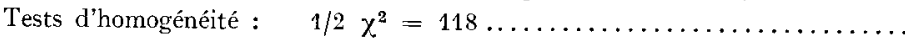

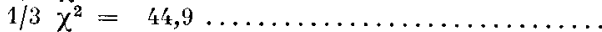

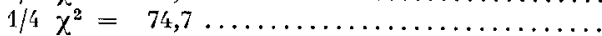

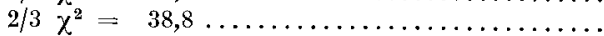

$2 / 4 \chi^{2}=64,7 \ldots \ldots \ldots \ldots \ldots \ldots \ldots \ldots \ldots \ldots$

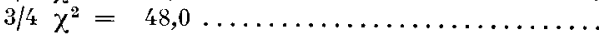

significatif

non significatif significatif

non significatif significatif non significatif 
mesure du pourcentage de rémission et celle du pouvoir de rétention d'eau et dans l'ordre des valeurs croissantes pour le $\mathrm{pH}$.

Dans le tableau 5, nous avons rassemblé les coefficients de corrélation par échantillon entre les quatre séries de mesures effectuées.

Les tests d'homogénéité des variances et des coefficients de corrélation ont été effectués selon SNEDECOR (I956).

Enfin, les tableaux 6 et 7 rassemblent, d'une part, les moyennes globales obtenues sur l'ensemble des échantillons et, d'autre part, les coefficients de corrélation totaux.

\section{TABLEAU 6}

Valeurs moyennes et écart-types globaux des déterminations effectuées sur l'ensemble des échantillons

\begin{tabular}{|c|c|c|}
\hline Variable & Moyenne $\bar{x}$ & Écart-type $\sigma$ \\
\hline pH muscle entier ... & 5,85 & 0,26 \\
\hline $\mathrm{pH}$ broyat...... & 5,84 & 0,26 \\
\hline Pourcentage de rémission. & 16,7 & 5,6 \\
\hline Pouvoir de rétention d'eau & 15,1 & 6,2 \\
\hline
\end{tabular}

TABLEAU 7

Coefficients de corrélation tolaux entre les 4 déterminations effectuées

\begin{tabular}{|c|c|c|c|}
\hline $\mathrm{pH}$ broyat...$\ldots \ldots \ldots$ & $+0,96\left({ }^{* *}\right)$ & & \\
\hline Pourcentage de rémission. & $-0,42\left(^{* *}\right)$ & $-0,42(* *)$ & \\
\hline \multirow[t]{2}{*}{ Pouvoir de rétention d'eau. } & $-0,58(* *)$ & $-0,59(* *)$ & $+0,28(* *)$ \\
\hline & $\begin{array}{l}\text { pH muscle } \\
\text { entier }\end{array}$ & $\mathrm{pH}$ broyat & $\begin{array}{l}\text { Pourcentage } \\
\text { de rémission }\end{array}$ \\
\hline
\end{tabular}

$\left(^{*}\right) \quad=$ significatif à $\mathrm{P}=0,01$

$\left({ }^{* *}\right)=$ significatif à $\mathrm{P}=0,05$

Lorsque 1'on classe les muscles par rapport aux différentes mesures effectuées, en considérant chacun des caractères ainsi mesurés comme critère de l'affection, on remarque que, sans être identiques, ces classements sont assez voisins, surtout en ce qui concerne les muscles classés en tête. Dans toute carcasse de porc, certains muscles sont donc toujours plus pâles; ils ont un pouvoir de rétention d'eau moindre et un $\mathrm{pH}$ ultime inférieur. Ces muscles sont les premiers à montrer l'existence de 1a myopathie exsudative et dépigmentaire et à présenter les défauts qu'elle entraîne. 
Ce sont essentiellement le muscle Longissimus dorsi et la majeure partie des muscles du membre postérieur.

Les caractères mesurés présentent une grande variabilité. On constate, en effet, en s'en tenant aux moyennes, que le pH varie de 5,43 (muscle Longissimus dorsi) à 6, I5 (Rectus femoris, partie foncée), le pourcentage de rémission de 3I,4 p. Ioo (muscle Semi tendinosus, partie claire) à II,3 p. Ioo (Plantaris) et le pouvoir de rétention d'eau de 27 p. roo (muscle Longissimus dorsi) à 7,4 p. Ioo (Pectoralis superficialis pars sterno costalis, partie claire). En considérant différents muscles d'un même porc, nous avons obtenu des valeurs extrêmes de 5,30 (muscle Longissimus dorsi) à 6,48 (Rectus femoris, partie foncée), pour le $\mathrm{pH}$, de 45 p. Ioo (muscle Semi membranosus) à II,5 p. Ioo (Plantaris) pour le pourcentage de rémission et de $42 \mathrm{p}$. Ioo (muscle Longissimus dorsi) à 7 p. Ioo (muscle Coraco-brachialis) pour le pouvoir de rétention d'eau. Entre deux portions d'un même muscle, nous avons obtenu des écarts également considérables; c'est ainsi que nous avons observé des valeurs de $\mathrm{pH}$ de 6,48 et 5,60 pour les deux parties du muscle Semi membranosus. De même, en ce qui concerne le pourcentage de rémission, des valeurs de I2,8 et 25,6 ont été obtenues sur le muscle Rectus femoris et, pour le pouvoir de rétention d'eau, I5,5 et 42,8 sur le muscle Semi tendinosus.

Les tests d'homogénéité des variances montrent que celles-ci sont significativement différentes.

L'examen des coefficients de variation montre que, pour une caractéristique musculaire donnée, ils sont, à de rares exceptions près, du même ordre de grandeur. Ceci signifie que, plus un muscle est pâle et a un pouvoir de rétention d'eau faible, plus ces caractéristiques ont une variabilité importante. Donc, lorsque l'on désire caractériser une carcasse par la détermination de l'une ou de plusieurs de ces caractéristiques, on a intérêt, dans le but d'augmenter la sensibilité de la mesure, à s'adresser aux muscles les plus atteints, que nous avons mentionnés précédemment.

En ce qui concerne les coefficients de corrélation entre les différentes caractéristiques mesurées, nous pouvons noter une variation assez importante d'un muscle à l'autre, à l'exception du coefficient de corrélation entre le $\mathrm{pH}$ mesuré sur échantillon entier et le $\mathrm{pH}$ mesuré sur broyat, ce qui est logique. On peut toutefois remarquer, dans ce dernier cas, que ce coefficient est nettement plus faible dans le cas du muscle Longissimus dorsi ; ceci est dû à la faible variabilité du $\mathrm{pH}$ de ce muscle, ce qui explique aussi les valeurs faibles pour ce muscle, des corrélations entre le pH (mesuré des deux manières) et le pouvoir de rétention d'eau ou le pourcentage de rémission. Par contre, la corrélation entre ces deux dernières caractéristiques est plus élevée et significative à $\mathrm{P}=0,05$.

Les tests d'homogénéité des coefficients de corrélation indiquent qu'il n'y a des différences significatives que pour la corrélation entre le $\mathrm{pH}$ mesuré sur muscle entier et le pH mesuré sur broyat et pour les corrélations entre le pouvoir de rétention d'eau et le $\mathrm{pH}$ (muscle entier et broyat). Dans le premier cas, ce résultat est probablement dû̀ à la valeur du coefficient pour le muscle Longissimus dorsi, très écartée de l'ensemble des valeurs obtenues pour les autres muscles. Par contre, pour les corrélations entre le pouvoir de rétention d'eau et le $\mathrm{pH}$, ce résultat indique que la liaison entre ces deux caractéristiques varie d'un muscle à l'autre. Cependant, la faible variabilité de certaines caractéristiques dans certains muscles peut expliquer la valeur basse des coefficients de corrélation obtenus pour certains échantillons; 
dans quelques cas, on doit plutôt invoquer la taille réduite ou la structure hétérogène des échantillons dans certains porcs, ce qui n'a pas permis d'effectuer les mesures avec toute la rigueur désirable.

Le reproche essentiel fait aux viandes exsudatives étant la faible valeur du pouvoir de rétention d'eau, nous avons pensé qu'il serait intéressant de voir comment se répartit pondéralement la musculature de porcs de qualité différente entre plusieurs classes de pouvoir de rétention d'eau.

Nous avons estimé que, en première approximation, on pourrait situer un seuil d'acceptabilité vers 22 à 23 p. Ioo de perte de poids de l'échantillon dans nos conditions expérimentales; nous avons donc défini 5 classes de pouvoir de rétention d'eau:

\begin{tabular}{|c|c|c|}
\hline \\
\hline \multicolumn{3}{|l|}{$\begin{array}{l}\text { P. IOO } \\
-\end{array}$} \\
\hline moins de & $7,5 \ldots \ldots$ & excellent \\
\hline 7,5 & $I_{5} \ldots \ldots \ldots$ & bon \\
\hline $\mathrm{I} 5$ & $22,5 \ldots \ldots \ldots$ & moyen, acceptable \\
\hline 2,5 & $\ldots \ldots$ & déficient \\
\hline plus & $30 \ldots \ldots$ & très déficient \\
\hline
\end{tabular}

La figure I montre la répartition pondérale entre ces classes des muscles de porcs de bonne et mauvaise qualité. Ces schémas indiquent que le porc A a $75 \mathrm{p}$. Ioo de sa musculature dans les classes "bon " et " excellent " et 25 p. Ioo dans la classe
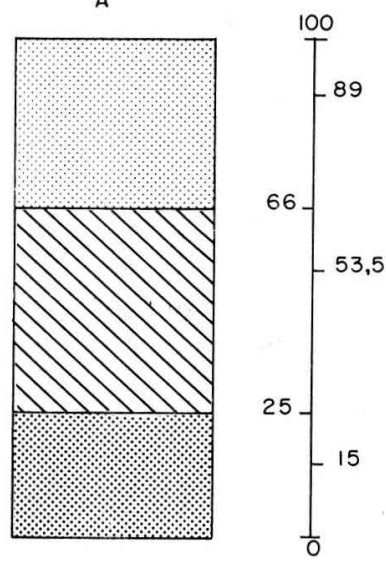

B

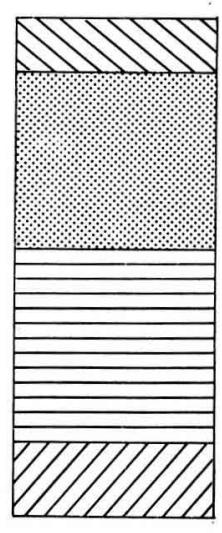

A bonne qualité B mauvaise qualité

$<<7,5 \%$

$\triangle .5$ à $15 \%$

\%०न 15 à $22,5 \%$

ए 22,5 a $30 \%$

ZIIZA $>30 \%$

FIG. I. - Répartition pondérale des muscles de porcs de qualité différente enire plusieurs classes de pouvoir de réteniton d'eau

acceptable ; le porc B, par contre, a plus de $50 \mathrm{p}$. Ioo de sa musculature dans les classes " déficient " et " très déficient ". Ceci montre les répercussions que peut avoir l'affection sur le plan économique, répercussions encore aggravées par le fait que les muscles qui se placent dans les classes de qualité inférieure sont essentiellement le muscle Longissimus dorsi et la majeure partie des muscles du membre postérieur et appartie anent donc aux morceaux de découpe ayant la valeur commerciale la plus élevée. 


\section{CONCLUSION}

L'étude de la répartition topographique de la myopathie exsudative et dépigmentaire du porc nous a montré qu'il existe toujours chez le porc des muscles présentant un $\mathrm{pH}$ peu élevé, une couleur pâle et un faible pouvoir de rétention d'eau par rapport à l'ensemble de la musculature. Ces muscles sont ceux qui sont atteints en premier lieu par l'affection et chez lesquels elle se manifeste avec le plus d'intensité. Ce sont donc ces muscles qui sont les plus intéressants à considérer pour diagnostiquer l'affection avec le maximum de précision et qui devront donc être utilisés pour l'étude de l'influence de divers facteurs sur le déterminisme de l'affection (régime alimentaire, environnement, etc.).

De plus, la mise en évidence d'une sensibilité propre à chaque muscle confère un intérêt particulier à l'étude ultérieure des diverses caractéristiques du muscle susceptibles d'être à l'origine de perturbations métaboliques dont les conséquences constituent l'étiologie même de l'affection. Parmi ces caractéristiques, il conviendrait de considérer la fréquence et l'intensité de l'activité du muscle, l'importance de son irrigation, ainsi que la nature de son équipement enzymatique, la concentration et 1'activité des enzymes de la glycolyse et de la chaîne respiratoire.

Reçu pour publication en juin 1966.

\section{REMERCIEMENTS}

Nous remercions l'Atelier mécanographique de la Station centrale de Génétique animale qui a effectué les calculs mécanographiques.

\section{SUMMARY}

TOPOGRAPHICAL DISTRIBUTION OF EXUDATIVE AND DEPIGMENTING MYOPATHY

(WATERY PORK) IN PIGS

To study the topographical distribution of exudative and depigmenting myopathy $3^{\circ}$ halfcarcases of Large White pigs killed at 100 $\pm 5 \mathrm{~kg}$ liveweight were dissected the day after slaughter. For each half-carcase $\mathrm{pH}$, percentage refiectance and water holding capacity were estimated in 5 I samples representing 45 muscles.

The samples were classed in relation to each of the characteristics measured (tables $1,2,3$ and 4 ). There was a certain similarity for those muscles at the top of the lists. That shows that crain muscles consistently have a lower final pH, a higher index of reflectance and a low capacity for retention of water. Moreover, in those muscles variation in the characteristics measured is greater. They are thus the best to consider when measurement of these characteristics is used for an objective assessment of the quality of the meat.

An examination of the weight distribution of the muscles of a half-carcase classed according to their capacity to retain water, corresponding to meats of different quality, shows the magnitude of the economic consequences of the disorder (figure I), particularly since the muscles most affected belong to the carcase cuts with the higher commercial values. 


\section{RÉFÉRENCES BIBLIOGRAPHIQUES}

Bendall J. R., LAwrie R. A., I964. Wässeriges Schweinefleisch. Eine Diskussion über Symptome und Ursachen. Fleischwirlschatt, 44, 41 I-42 I.

BriskEY E. J., I 964 . Etiological status and associated studies on pale, soft, exudative porcine musculature. Adv. Food Res., 13, 89-1 78 .

COSSARD J., I957. La myopathie exsudative el dépigmentaire du porc. 62 p. Thése Doct. Vétérinaire, Paris.

Goutefongea R., I966. Étude comparative de différentes méthodes de mesure du pouvoir de rétention d'eau de la viande de porc. Ann. Zootech. (sous presse).

Goutefongea R., 1963. Les viandes exsudatives. Ann. Zootech., 12, 297-337.

Mesle L., Charpentier J., Goutefongea R., Dumont B. L., ig6o. Note sur la variation des caractéristiques physicochimiques de la musculature du membre postérieur du porc. Sixth Meeting Meat Res., Insitul., Utrecht.

Nickel R., Schummer A., Serferle E., 1954. Lehrbuch der Anatomie der Haustiere. Band I, 502 p., Paul Parey, Berlin.

SNedecor G. W., I956. Statistical Methods, p. I 78 et $285.5^{\mathrm{e}}$ edition. The Iowa State University Press, Ames, Iowa. U. S. A.

ANNEXE

Liste des muscles disséqués

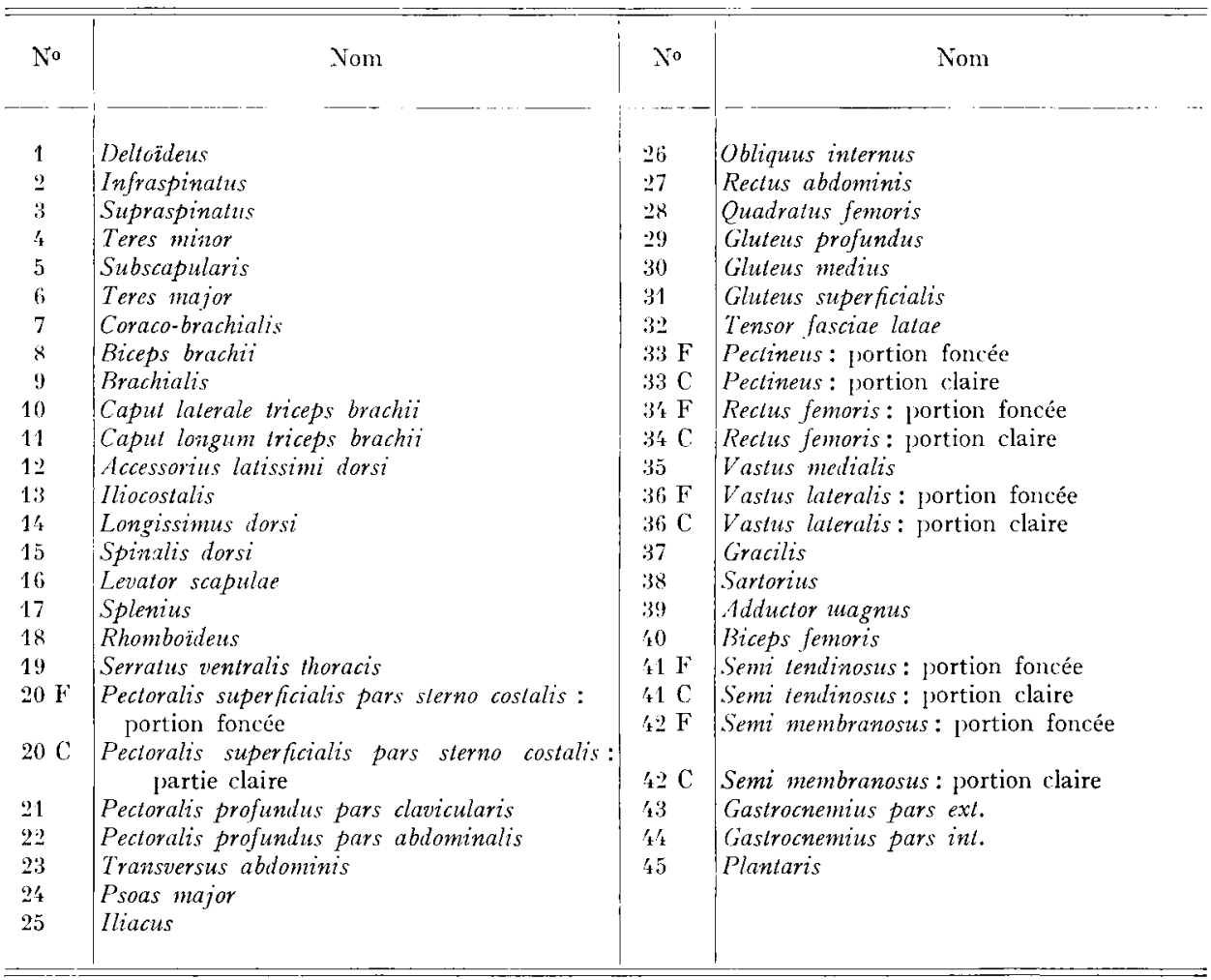

